\title{
OUTSTANDING THEORETICAL PROBLEMS
}

\author{
C.J. DURRANT \\ Department of Applied Mathematics \\ University of Sydney \\ NSW 2006 \\ Australia
}

\begin{abstract}
The development of model atmospheres from the 'classical'static but deductive models to present-day dynamic but inductive models is sketched. The main problems facing theory are defined in terms of the need to produce a post-classical inductive model. Attention is focused on the most promising tool available today, the computers able to realize simulations of astrophysical systems. The direction of progress in the areas of radiative transfer, convective transport, waves and oscillations, and MHD is reviewed. It is concluded that the major outstanding radiative and hydrodynamic problems are likely to be elucidated in the foreseeable future, especially if there is a suitable commitment by the international community. However, the understanding of the behaviour of magnetic fields and their associated activity will require a longer, but no less urgent, programme.
\end{abstract}

\section{Introduction}

It is a curious fact that the first recorded use in the English language of the word 'atmosphere' is a reference in 1638 to the vaporous surroundings of the Moon! But by 1677 the word had gained its commonest connotation, that of the body of air around the Earth. To most people today, the atmosphere means the terrestrial atmosphere, not that of the Sun.

Early last year there was a meeting in Canberra of the IGBP, the International GeosphereBiosphere Program. The aim of this program is to 'describe and understand the interactive physical, chemical and biological processes that regulate the total Earth system'. It is of course the impact of human society on the terrestrial environment which has given the IGBP its impetus and its urgency. Not much interest was displayed at that meeting in the Sun, although it will have an inexorable effect on human society in the long term if not the short.

Fortunately, the influence of human activity does not yet extend to the Sun, but many of the physical processes operating in the atmosphere of the Earth operate also in the atmosphere of the Sun. Whilst there is already a great body of knowledge about these processes, they 'are so highly interactive [in the terrestrial context] that an adequate quantitative synthesis of them is essential' (Tucker (1988)). The real challenge of the Program is to make the synthesis quantitative.

Exactly the same comments apply to the current situation in solar physics. If we test our understanding by demanding that we should be able to make quantitatively accurate predictions of the behaviour of the atmospheres of other stars, then we would have to admit that we have not advanced very far in the last 70 years.

\section{9}

J. O. Stenflo (ed.), Solar Photosphere: Structure, Convection, and Magnetic Fields, 489-499. (c) 1990 by the IAU. 
Theoretical foundations were laid around the turn of the century with the work of Lane (leading up to Emden) on equilibrium stratification, of Schwarzschild on radiative equilibrium and of Milne on local thermodynamic equilibrium. Over the following 60 years the equations governing these three processes allowed the construction of model photospheres which increasingly resembled the empirical one as the frequency distribution of the opacity was treated more accurately (Kurucz (1979)). I shall refer to these as 'classical' models.

As a simple model of the photosphere, the classical model is quite impressive. Moreover, the calculations can be extended to any other star (Kurucz (1979)) and so our understanding of the atmosphere at this level meets our practical test.

Unfortunately, this is not quite true. If the assumed equilibria are tested for physical consistency throughout the model, the criterion developed by Schwarzschild himself reveals that hydrostatic and radiative equilibrium cannot be sustained in and below the continuum levels. A new process, that of convection, had to be introduced. To cope with the modifications to the energy and momentum balance resulting from the convective motions, an aerodynamic description of the process was developed by Biermann and Siedentopf in the early 1930s. This changed the nature of the model of the solar atmosphere. Before, it had been built on knowledge and insight about the system of concern; with the adoption of the mixing-length description of turbulent convection, the model had to draw upon observations. It required the empirical determination of parameters and drew heavily on laboratory experiments with turbulence. The models changed from being 'deductive' to 'inductive' (Karplus (1977)).

In practice this has meant that all solar models now contain adjustable parameters of one sort or another. To quote Tucker (1988) again, 'empiricism is required to develop analogs for some component (processes) and to generally "tune" the models, sometimes in an arcane way'. The removal of the adjustable components is an outstanding problem of theory.

We are more fortunate than the IGBP modellers in that we can write down the equations that govern the behaviour of stellar photospheres. The fluid equations and radiative transfer equations can be used (with justification) below the level of the transition region. Kinetic effects can be produced in the electron distribution in the non-LTE regions of the chromosphere (Shoub (1977)) but any dynamical consequences are not of immediate concern in the photosphere. The classical model was a consistent solution of these governing equations in static equilibrium. The convective models were not consistent solutions because the actual terms in the equations were replaced by model terms constructed from empirical knowledge. We have still to understand what the full equations will yield under circumstances appropriate to the Sun.

I must stress here that at least as important as the equations themselves are the boundary conditions and on this point I echo the concern expressed by Thomas (1983) about treating model systems as isolated systems. The construction of physically consistent boundary conditions is part of the theoretical task. It does not take us outside current physical theory. I confess to being in Thomas' words a 'speculative theorist'. We should understand what that theory really tells us about stellar atmospheres before appealing to new physics.

\section{The way ahead}

Let us look first at the tools at our disposal. Since direct experiment is impossible, we have to pursue indirect investigations. Tucker (1988) enumerates the possibilities: 
1. mathematical analysis of the governing equations,

2. experiment with miniature analogue systems,

3. experiment with numerical simulation.

Analysis is the classical avenue but it has proved inadequate as far as the full set of equations is concerned. However, analysis has produced an enormous corpus of knowledge about individual processes, gained by isolating and adjusting terms (i.e. the physical interactions) until a mathematical system of sufficient simplicity emerges. Truesdell (1980) claims that this style of investigation originated in the study of thermodynamics in the 19 th Century. It results in what can be called 'scenario physics'. If the scenario could be realized, we would understand the physics. But scenarios are generally competitive, what one assumes is contradicted by another. In the Sun, the processes are mixed and modified by their interaction. To understand the Sun, we have simply got to address this problem.

Some of the consequences of the interactions, certainly more than have been elucidated by analysis, can be demonstrated by the second tool, analogue experiments. However, because laboratory circumstances are so different from those in the solar atmosphere these experiments are rarely of direct relevance. Outstanding exceptions are the 'ice-water' experiment of Townsend (1964), which produces convective overshoot, and the Spacelab 3 experiment which produces convection in a rotating, stratified medium (Hart et al. (1986)). Although limited in number and scope, such experiments are the only means by which the ideas that guide the development of scenario physics can be tested in a controlled manner. I hope that ingenuity will continue to be expended on devising more of these experiments.

However, I think that the means of breaking out of the straightjacket of the past few decades in provided by the third avenue, the development of the high-speed computer. It is the only tool that we possess that can in principle bridge the gap between the laboratory and the Sun. Since 1940 each decade has seen a phenomenal growth in the power of computers, about half due to improvements in hardware and about half due to improvements in software. Computer manufacturers claim that this trend will continue over the next decade and are confident that massively parallel systems will maintain the momentum into the decades beyond. In 1984 Nordlund wrote that detailed numerical simulations using the full set of magnetohydrodynamic equations were '(at least marginally) feasible with present day computers'. It will clearly not be long before the cautious qualification can be removed.

I should like to stress, though, that numerical simulation is a form of experimentation. The numbers obtained should be regarded as the outcome of an experiment, an experiment designed to demonstrate physical processes that require to be understood. This has two consequences. Firstly, if the result is not interpreted in a manner that increases our physical understanding, the experiment has no value. Here scenario physics finds a legitimate rationale. It provides the building blocks of cause and effect which allow the results to be expressed in terms of physical processes. The simulation sifts out the relevant scenarios from the irrelevant. An admirable example of this approach is set by the work of Nordlund $(1984,1985 \mathrm{a}, 1985 \mathrm{~b})$, in which close attention is given to elucidating the physical processes underlying the model results.

However, there is a step beyond this. As the theoretical jigsaw starts to fall into place, we should begin to recognize the quantitative importance of the interrelationships so that the full-scale model can be broken up into quasi-independent compartments. This is precisely the opposite direction to the analytical path in which the parts are studied before the whole. Only when we know to what extent the compartments influence one another, can 
we construct simple self-consistent models of the solar atmosphere beyond the classical level. Only then can the resulting theoretical structure be applied with confidence to stars other than the Sun. Only then will discrepancies really point to 'new physics'.

The second consequence of the experimental nature of simulation is the need for appropriate attention to the experimental procedure. Experiments should be reproducible and should be reported in sufficient detail to allow them to be reproduced. This requires specification of

1. the governing equations,

2. the algorithm used to solve them,

3. the physical boundary conditions,

4. the implementation of the boundary conditions.

The major area of uncertainty in astrophysical simulations lies in the boundary conditions at the inner and outer limits of resolution. I shall return to this subject below.

To illustrate these points I want to look briefly at four areas of solar physics in the order in which I believe progress will be made, radiative transfer, convective transport, overshoot and waves, and MHD.

\section{Radiative transfer}

In this case both the governing equations and appropriate upper and lower boundary conditions are well established. The development of theories of radiative energy transport has differed essentially from that of hydrodynamic transport in that the nonlocal nature of the process was recognized from the outset. The radiation field is obtained by solving selfconsistently along all rays, taking explicit account of any inhomogeneity and anisotropy of the system.

Faced with this need, a great deal of effort has been expended on devising efficient algorithms and ones which exploit the ability to do parallel computations along different rays at different frequencies. These are the subject of a recent monograph (Kalkofen (1987)). The need to incorporate a realistic model of the 'microphysics' has also been accepted and calculations can be made using non-LTE where necessary.

The problem of resolution has already been mentioned and it has been overcome, as computing resources have improved, by increasing the number of points at which the frequency is sampled. Indeed, at this point the subject has progressed to the stage of looking for recipes for simpler models that capture the behaviour established in more detailed calculations. The representation of the opacity distribution in frequency by statistical sampling or distribution functions (Kurucz (1979), Carbon (1979)) is a start in this direction. Nordlund (1982) has suggested source function averaging.

I would suggest that there are no outstanding theoretical problems as far as the radiation physics of the photosphere is concerned, though the treatment of non-LTE transfer in rapidly moving structures in the chromosphere may produce significant effects of some subtlety which do not form a part of our intuitive thinking at present. The accuracy of transfer calculations is generally limited though by the desperate shortage of accurate atomic data, particularly of collisional cross sections. 


\section{Convective transport}

Convection is the next most important ingredient in the photospheric system. It governs the mean structure of the lowest levels and modulates that above. It also underlies, in one way or another, the entire horizontal structuring of the photosphere (a classical static atmosphere has no such structure).

Here the governing equations are well known. The anelastic approximation has been extensively used (e.g. Nordlund (1982 et seq.)) because it allows an explicit algorithm to be used to study convective motions without inducing sound waves. However, efficient implicit schemes now allow the full compressible equations to be solved (Chan and Sofia (1986)). This becomes essential when the magnetic field is included.

Also well known are the various processes implicit in the equations-buoyancy, pressure gradients, radiative 'conduction', kinetic energy transfer by the nonlinear inertial term and viscous dissipation. But we do not understand how they interact to determine the structure of convective flow in a highly stratified turbulent medium like the outer parts of the solar convection zone. Computer simulations are already proving their value as an experimental tool. Progress is not direct because no computer can (or is likely to) encompass the range of scales from global $\left(10^{8} \mathrm{~m}\right)$ down to viscous (less than a metre).

Moreover, the computation cannot be simplified by reducing the number of dimensions. Astrophysical convection is turbulent and is thus an essentially three-dimensional phenomenon. Artificial two-dimensional models have quite different characteristics-a cascade of energy to large scales, for instance. Two-dimensional simulations are no substitute for three-dimensional simulations. With hindsight and a secure knowledge of the threedimensional system we may at some future stage be able to justify the use of models of lower dimension for some purposes, but not at present.

Since we are interested primarily in scales in the range $10^{5}-10^{7} \mathrm{~m}$ our models must account for the fact that the largest and smallest scales are not present. Periodic boundary conditions in the horizontal plane ensure that the model is embedded in a larger system and that there is no special treatment of side walls that might dictate the geometry of the flow. A cartesian geometry then guarantees that there is statistical uniformity and isotropy in the horizontal plane. Simulations in cylindrical geometry (e.g. Steffen (1987)) cannot avoid introducing anisotropy.

The upper boundary condition will be discussed in the next section. The lower boundary conditions are at present problematical. They must be set within the convection zone as there is no hope of extending a surface simulation down to the bottom. The solution adopted by Nordlund and Steffen is to allow 'free' flow into and out of the boundary, i.e. the vertical velocity gradient and the pressure fluctuation are set to zero. In each case the model flows stream in and out of the lower boundary and show no signs of closing. Thus the properties of the system outside the model should control the true behaviour of the flow just as much as those inside. Yet the model flow is determined entirely by the internal properties. The dilemma might be resolved if the vertical flows were an artifact of the 'free' boundary condition having no dynamical significance, in which case a boundary condition enforcing a circulation (e.g. the vertical velocity being set to zero) would have little effect over most of the box. Alternatively, the flow might be localled controlled at the level of the lower boundary, somewhat in the spirit of mixing-length formulations. Or any resemblence between the isolated model results and the behaviour shown by the same model embedded in a much larger system may be fortuitous. 
The answer can be provided definitively only by looking at the embedding problem more carefully. Here is there is a long-outstanding theoretical problem. Despite its obvious fundamental importance, even the basic form of convection in a stratified medium is not yet known. To date there are just two three-dimensional simulations-that of Graham (1977) and that of Chan and Sofia (1986). In the former the dominant mode is a quasi-cellular pattern extending the whole depth of the unstable layer whilst in the latter the vertical velocity structure loses its coherence over a pressure scale height. The models differ in many respects. Graham's model had a low Rayleigh number (ten times the critical value) and a Prandtl number around unity so that the flow was just turbulent. The degree of stratification was also small, just over one pressure scale height. The models of Chan and Sofia were more highly turbulent (only an effective Reynolds number of 300 is quoted) and extend over 4-5 scale heights. Because of this, Chan and Sofia had to model the scales below the level of numerical resolution, the so-called subgrid scales.

The standard treatment is one adopted by terrestrial modellers, in which it is assumed that the smallest resolved scale lies within the inertial regime of the turbulence where there is neither significant driving nor dissipation only a transfer of kinetic energy from large scales to small scales via the nonlinear inertial term in the equation of motion. The plausible assumption of isotropy and homogeneity then yields a Kolmogorov spectrum. The contributions of the unresolved motions to the momentum and energy equations can then evaluated as an eddy viscosity and an eddy conductivity (Rogallo and Moin (1984), Eidson (1985)). This formulation has been tested for incompressible turbulent flows for low Reynolds number $R e \leq 40$ by Clark et al. (1979). For compressible flow, most authors (e.g. Hurlburt et al. (1984)) model the subgrid heat diffusion as

$$
\langle K \nabla T\rangle_{s g}=\frac{\mu}{P r_{t}} \nabla T
$$

where $\mu$ is the subgrid eddy viscosity and $P r_{t}$ is the turbulent Prandtl number, taken to be about $\frac{1}{3}$. Chan and Sofia (1986) argue that in a stratified fluid it is the entropy gradient which drives the subgrid diffusion

$$
\langle K \nabla T\rangle_{s g}=\frac{\mu}{P r_{t}}\left[\nabla T-\nabla_{a d}\left(\frac{T}{\rho}\right) \nabla p\right]=\frac{\mu}{P r_{t}} \nabla S,
$$

the second term arising from the adiabatic gradient $\nabla_{a d}$. Since the entropy varies much less strongly than the temperature across a stratified convective layer, the subgrid heat transport is much smaller than previously estimated. The resolved motions are therefore more vigorous and appear to break up in a statistical sense over a vertical scale height.

Chan and Sofia describe careful tests of their algorithm and the parametrization of their model. Some unanswered questions remain though.

1. Their choice of upper and lower boundary condition reflects acoustic waves. A transmitting boundary would be more appropriate.

2. The rigid upper and lower boundaries also force the heat in their immediate neighbourhood to be carried by the subgrid motions. Yet the basis for their treatment breaks down when isotropy is broken by the influence of the boundary (Eidson (1985)). A narrow stable layer added at the boundaries would remove the possibility that unrealistic boundary effects propagate into the bulk of the system. They would also facilitate the implementation of transmitting boundary conditions. 
3. Does the numerical resolution really reach the inertial range so that the subgrid eddy viscosity is a valid estimate? A great deal of progress has been made recently in deriving parameter-free closures in statistical turbulence theory. Yoshikawa (1988a) offers a method that can yield subgrid models that take account of buoyancy. He finds that the turbulent Prandtl number (for passive scalar diffusion) is not constant but varies with the square of the ratio of the velocity to scalar variance dissipation times. Subgrid models may be tested against full resolved simulations of Rayleigh-Bénard convection up to Rayleigh numbers of $4 \times 10^{5}$ (Grötzbach 1982).

4. Finally, the way in which the models are discussed needs to be systematized. The flow visualizations, i.e. instantaneous plots of velocity vectors, tend to focus attention on the coherent motions, whilst spatially averaged cross correlations between the velocities at different vertical heights reveal that much of the motion is disordered. Clearly, convective plumes of limited spatial extent coexist with random motions. We really need to know how much heat is carried in each, and this requires more sophisticated statistical methods. Adrian (1977) gives a full discussion of how higherorder moments and conditionally averaged statistics can be used to interpret coherent structuring in turbulent convective flows and Kerr (1985) describes skewness and kurtosis factors in numerical turbulence simulations.

I think the organization of motions in stellar convection zones is close to being a solved problem. We would then be able to investigate one of the most enigmatic features of the photosphere, the supergranulation. Nordlund (1985) has suggested how the model might be set up; it should decide whether or not the supergranulation is a direct manifestation of convection zone dynamics.

\section{Overshoot and waves}

Overshoot has been the subject of the most detailed dynamical modelling to date. Nordlund (1982 et seq.) has elucidated many of the properties of the granulation by this means. In particular, an understanding of the heat exchange between the flowing gas and the radiation field emerged only from these simulations. On average heat is extracted from the radiation field to heat the gas which cools as it expands into the stably stratified region. The mean temperature of the atmosphere is depressed below the radiative equilibrium values and the model closely matches the empirical structure.

Better knowledge of the convection zone dynamics would allow the lower boundary condition described above to be improved. The upper boundary condition can be formulated in some detail by fitting appropriate wave-like solutions in the stable region. Nordlund (1982) found this unnecessary for anelastic modelling but will be more important for fully compressible models.

A fully compressible treatment is the next step and will yield the first reliable estimates of the acoustic energy flux produced in the upper parts of the solar convection zone. These will provide a crucial test of our ideas concerning the mechanical heating of the solar chromosphere.

Global acoustic modes will be modified or suppressed by finite box models so any coupling between oscillations and convection would be revealed only by an iteration between global scale simulations with parametrized convective scales and convection models with impressed global motions. 
Convective motions overshooting into stable layers distort the gravitational equipotential surfaces and generate internal gravity waves in laboratory fluids. However, solar observations show no strong gravity wave field despite considerable convective overshooting in the granulation. Nordlund has explained their absence in terms of his model. Convective pressure disturbances can penetrate far in a stable layer of decreasing mean pressure and drive an extended circulation rather than an oscillation in the photosphere. Gravity waves can only be excited higher in the atmosphere.

The most uncertain aspect of photospheric dynamical modelling must lie in the subgrid scale motion. Nordlund (1982) modelled the subgrid viscosity heuristically. In reality, the transition from turbulent transport processes in the convective layer to wave transport in the stable is likely to be complicated. Fernando (1988) studied the development of turbulence in a stably stratified layer in the laboratory. A locally generated patch of turbulence grows by entrainment of nonturbulent material until the vertical mixing is limited by negative buoyancy forces. Thereafter an interfacial layer develops separating the turbulent region from the overlying nonturbulent material. Turbulent disturbances at the interfacial layer generate internal waves above. As the waves break small regions of turbulence are created above the interface which eventually merge and are incorporated by the turbulent patch, thereby slowly raising the interface.

\section{MHD}

The incorporation of the magnetic field into the hydrodynamic system is without doubt the most important outstanding theoretical problem. Without it we cannot answer the questions

1. Does the hydrodynamic convection organize the field on the granular and supergranular scales or might the field organize the structure on the supergranular scale or is the structure due to a subtle process of self-organization?

2. What is the structure of the field in the photosphere, why is there strong field and weak field? Where do the electric currents flow and what is their strength?

3. What is the spectrum of dynamic disturbances exhibited by the photospheric field?

These are the questions crucial to understanding the whole atmosphere of the Sun and other stars above the photosphere. The dynamic behaviour of the field and current system in the photosphere and below provides the driver in electrodynamic theories of the outer layers of stars.

Simulations such as those of Nordlund in my opinion now provide more ccurate estimates of the spectrum of granular dynamics, the root-mean-square values being reported as

$$
\left\langle v_{z}\right\rangle=2 \mathrm{~km} \mathrm{~s}^{-1}, \quad\left\langle v_{h}\right\rangle=3 \mathrm{~km} \mathrm{~s}^{-1}, \quad\langle\delta p / p\rangle=0.5 .
$$

These are much higher than the observationally derived values, which still suffer from lack of spatial resolution and ambiguity of interpretation. Since the study of magnetic field structures requires even higher resolution and more complicated diagnostics, experiment seems unlikely to provide meaningful information of the current system (for which gradients of field strength must be measured) or of dynamical disturbances (for which the distortion of the field structure must be measured). Numerical simulations seem to offer the only hope of substantial progress here. 
Nevertheless, the theoretical problems are immense. The grid size required to resolve a magnetic field element adequately is so small that the size of the simulation box must be reduced correspondingly. Subgrid modelling is complicated by the fact that nonlinear effects can operate either from large scales to small-like the hydrodynamic kinetic energy cascadeor from small to large in an inverse cascade. Yoshizawa (1988b) has recently proposed a subgrid model for MHD turbulence based on statistical theory. However, looking at fully resolved hydrodynamic (Kerr (1985)) and MHD (Meneguzzi et al. (1981)) simulations at quite moderate Reynolds number $(R e \leq 100, R m \leq 100)$ for fluids with $\operatorname{Pr} \sim 1$, one is struck by the spatial intermittency of the vorticity and magnetic field on the smallest scales. Moreover, Meneguzzi et al. point out that the field concentrations do not coincide with the vorticity concentrations. It is perhaps well to remember that statistical ensembles may not model an individual subgrid volume, even when spatially averaged.

The problem of modelling the embedding of the box in physically realistic surroundings is also that much greater. This difficulty is compounded by the fact that the magnetic field cannot be isolated, even conceptually, within the box as can, for example, an eddy of the flow. The field must leave the box at top and bottom so that we require magnetic boundary conditions. These are best thought of in terms of the equivalent boundary conditions on the current system. Where there is field at the boundary, mass flow and current flow are ducted in an out of the box. Clearly these are no locally determined properties of the system. They adjust in response to the need to evolve the whole magnetic structure.

At the upper boundary it may be possible to establish a form of equivalent circuit for the coronal connections using the theory of Ionson (1982). At the lower boundary the circuit cannot be continued. The structure of the field elements below the surface is unknown-do they collect together in larger units or are they uniformly dispersed? An iteration between high-resolution and low-resolution models, as in the case of convection modelling, might yield a heuristic solution but its implementation seems a long way off.

\section{Prospects}

In summary, I think the prospects for solving the problems of the hydrodynamic behaviour of the solar photosphere are good. We are almost at the stage where the structure and dynamical effects in a stellar atmosphere can be calculated without recourse to empirical scalings. It remains to be seen, though, to what extent the physical insight provided by complex simulations allows the computations to be simplified. On the other hand, the continued increase in computational power might overtake the need to simplify substantially. Within a decade we may all be able to run off the models on cheap massively parallel computers. In either case, present work should have physical insight as its main aim.

Of course, the immediate future lies with big and expensive machines, available only to few. The time seems ripe for an organized international effort dedicated to astrophysical convection simulations, perhaps along the lines of the summer program at the Center for Turbulence Research at NASA Ames Research Center and Stanford University (Hunt (1988)).

But we should be also be planning the attack on the MHD problem. To return once again to the IGBP. Its ambitious program will cover at least a decade starting in 1990 . However, little interest was shown at the Canberra meeting in the influence of the Sun on the variability of the Earth system in either the short term or the long term. In the short 
term, it is solar magnetic activity which affects us on Earth the most. The IGBP will not be complete without an understanding of the MHD behaviour of the Sun. This is controlled in no small part by the processes at the surface and in the immediate subsurface layers. The understanding of these is the outstanding theoretical problem of the next decade.

\section{References}

Adrian, R.J. (1977) Journal of Fluid Mechanics, 69, 753-781.

Carbon, D.F. (1979) Annual Review of Astronomy and Astrophysics, 17, 513-549.

Chan, K.L. and Sofia, S. (1986) Astrophysical Journal, 307, 222-241.

Clark, R.A., Ferziger, J.H. and Reynolds, W.C. (1979) Journal of Fluid Mechanics, 91, 1-16.

Eidson, T.M. (1985) Journal of Fluid Mechanics, 158, 245-268.

Fernando, H.J.S. (1988) Journal of Fluid Mechanics, 190, 55-70.

Graham, E. (1977) In E.A. Spiegel and J.P. Zahn (eds.), Problems of Stellar Convection, Springer-Verlag, Berlin, pp. 151-155.

Grötzbach, G. (1982) Journal of Fluid Mechanics, 119, 27-53.

Hart, J.E., Glatzmeier, G.A. and Toomre, J. (1986) Journal of Fluid Mechanics, 173, 519-544.

Hunt, J.C.R. (1988) Journal of Fluid Mechanics, 190, 375-392.

Hurlburt, N.E., Toomre, J. and Massaguer, J.M. (1984) Astrophysical Journal, 282, 557-573.

Ionson, J.A. (1982) Astrophysical Journal, 254, 318-334.

Kalkofen, W. (ed.) (1987) Numerical Radiative Transfer, Cambridge University Press, Cambridge.

Karplus, W.J. (1977) Mathematics and Computers in Simulation, 19, 3-10.

Kerr, R.M. (1985) Journal of Fluid Mechanics, 153, 31-58.

Kurucz, R. (1974) Astrophysical Journal Supplement, 40, 1-340.

Meneguzzi, M., Frisch, U. and Pouquet, A. (1981) Physical Review Letters, 47, 10601064.

Nordlund, А. (1982) Astronomy and Astrophysics, 107, 1-10.

Nordlund, A. (1984) In S.L. Keil (ed.), Small-Scale Dynamical Processes in Quiet Stellar Atmospheres, Sacramento Peak Observatory, Sunspot, pp. 181-221.

Nordlund, A. (1985a) In H.U. Schmidt (ed.), Theoretical Problems in High Resolution Solar Physics, Max-Planck-Institut für. Astrophysik, München, pp. 1-24 and pp. 101119.

Nordlund, A. (1985b) Solar Physics, 100, 209-235.

Rogallo, R.S. and Moin, P. (1984) Annual Review of Fluid Dynamics, 16, 99-137.

Shoub, E.C. (1977) Astrophysical Journal Supplement, 34, 259-275. 
Steffen, M. (1987) In E.-H. Schröter, M. Vázquez and A.A. Wyller (eds.), The Role of Fine-Scale Magnetic Fields on the Structure of the Solar Atmosphere, Cambridge University Press, Cambridge, pp. 47-52.

Thomas, R.N. (1983) Stellar Atmospheric Structural Patterns, NASA SP-471, CNRS and NASA, Paris and Washington.

Townsend, A.A. (1964) Quarterly Journal of the Royal Meteorological Society, 90, 248259.

Truesdell, C. (1980) The Tragicomical History of Thermodynamics 1822-1854, SpringerVerlag, New York.

Tucker, G.B. (1988) In Global Change, Australian Academy of Science, Canberra, pp. 182-191.

Yoshizawa, A. (1988a) Journal of Fluid Mechanics, 195, 541-55

Yoshizawa, A. (1988b) Physics of Fluids, 30, 1089-1095. 